\title{
Development of Wearable Temperature Sensor Based on Peltier Thermoelectric Device to Change Human Body Temperature
}

\author{
Eun-bin Park, ${ }^{1}$ Seyed Jamaleddin Mostafavi Yazdi, ${ }^{2}$ and Jong-Ha Lee ${ }^{1 *}$ \\ ${ }^{1}$ Department of Biomedical Engineering, School of Medicine, Keimyung University, 42601 Daegu, South Korea \\ ${ }^{2} \mathrm{NVH}$ and Experimental Mechanics Laboratory, Department of Mechanical Engineering, \\ Kettering University, 1700 University Ave, Flint, MI 48504, USA
}

(Received December 16, 2019; accepted May 13, 2020)

Keywords: wearable, temperature sensor, thermoregulation, feedback control system, Peltier

We demonstrate a wearable temperature sensor based on a Peltier thermoelectric device. The wearable temperature sensor is attached to the wrist using a bracelet, which can monitor, control, and change the human body temperature using a local temperature change. The body temperature was changed by transferring the heat from one side to the other side of the Peltier semiconductor or vice versa. The feedback control system was used to maintain the human body temperature during increases and decreases in the temperature of the surrounding environment. The results showed the changes in body temperature over $300 \mathrm{~s}$ in 100 trials with different rates of increase and decrease in the temperature. The highest relative increase in temperature was $86.1 \%$ with a change from 18.7 to $34.8{ }^{\circ} \mathrm{C}$, while the lowest relative increase was $1.2 \%$ with a change from 24.6 to $24.9{ }^{\circ} \mathrm{C}$. The average change was $33.5 \%$ with a standard deviation of $25.5 \%$. This technology provides a basis for next-generation wearable temperature sensors to control and change the temperature of the human body.

\section{Introduction}

\subsection{Body temperature}

The body temperature of a human or animal is maintained by the heat energy provided by burning nutrients during cellular oxidative metabolism. To maintain the temperature of the human body at $36-37^{\circ} \mathrm{C}$, the heat generated and lost should be balanced. ${ }^{(1)}$ Therefore, thermoregulation plays a key role in maintaining the body temperature.

The body temperature includes core and skin temperatures. The core temperature is defined as the body temperature in daily life. There are several ways to measure the core temperature, including esophageal, tympanic, oral, and rectal measurement, where rectal measurement is the most accurate. Environmental conditions and blood temperature affect the measurement of oral and esophageal temperatures, respectively. The tympanic temperature is equal to the temperature of the hypothalamus, and it reflects the concentration of blood going

${ }^{*}$ Corresponding author: e-mail: segeberg@kmu.ac.kr https://doi.org/10.18494/SAM.2020.2741

Acknowledgments were revised on October 27, 2020 [refer to errata in Vol. 32, No. 10(3)]. 
to the thermoregulatory center. The skin temperature is typically $5-8{ }^{\circ} \mathrm{C}$ less than the core temperature and depends on the location of the skin. The lowest temperature of the skin is at the fingers, toes, ears, and the tip of the nose, while the highest temperature is at the forehead and trunk. Two factors significantly affect the skin temperature: the environment and the type of skin. The skin temperature increases or decreases during sweating owing to water evaporation.

There are several factors affecting the body temperature, including age, hormones, stress, surgery, and body movement. The body temperature also varies with the outside temperature via the thermoregulatory center, which develops within six months of birth. The level of thermoregulation at the age of 10 is similar to that of an adult and decreases with age. The stimulation of nerves and hormones by stress increases the metabolic rate, which may increase the body temperature. In addition, movement increases the metabolic rate and heat production. The total amount of heat produced by the body depends on the intensity and length of the movement, as well as the ambient temperature. Figure 1 illustrates the balance between heat generation and loss in the human body.

\subsection{Importance of body temperature}

Maintaining the body temperature at $37{ }^{\circ} \mathrm{C}$ is vital for the human body and it boosts the immune system as well as the circulation of blood flow to cope with any diseases. When the body temperature decreases by $1{ }^{\circ} \mathrm{C}$, the immunity decreases by $30 \%$. Therefore, a decrease in body temperature can increase the risk of developing viral diseases. Moreover, hypothermia occurs as the body temperature drops below $35^{\circ} \mathrm{C}$, which can cause cardiac arrest and reduced hormone secretion, overall muscle movement, and metabolic ability. Figure 2 shows the physiological effect of different body temperatures. If there is abnormal thermoregulation of the human body, it can be controlled by wearable devices such as a wearable temperature sensor. Regulation of the local body temperature is used to control the whole-body temperature. When the local temperature rises via a local thermal effect, the metabolism increases energy consumption and generates heat. The temperature is increased by $10{ }^{\circ} \mathrm{C}$ when the metabolism

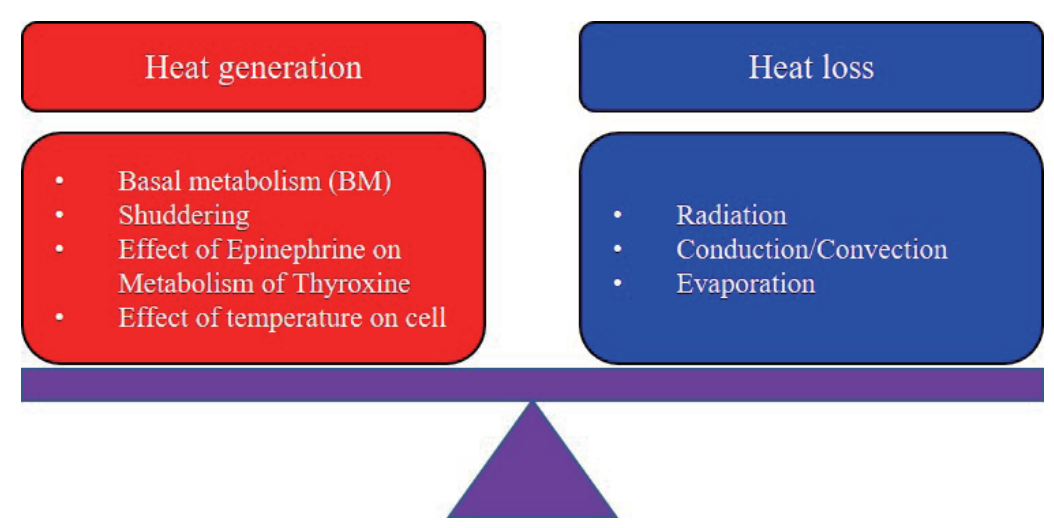

Fig. 1. (Color online) Balance between heat generation and heat loss in the human body. 


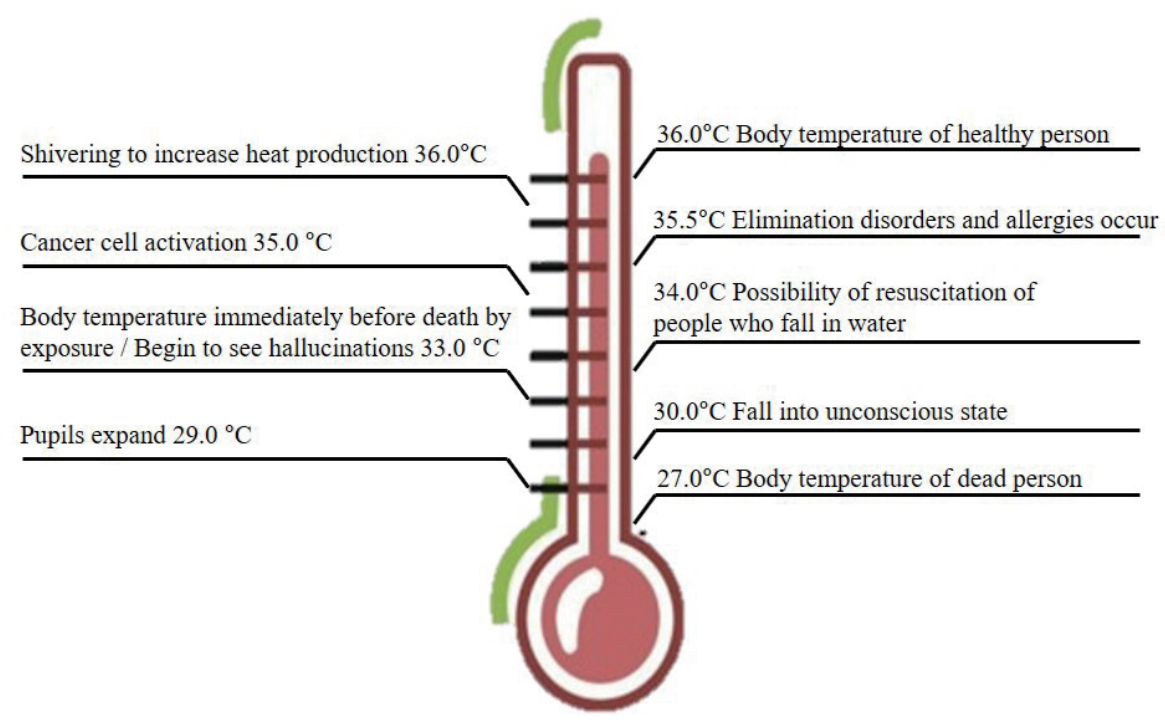

Fig. 2. (Color online) Physiological effects of different body temperatures.

is increased 2.5 -fold according to van't Hoff's law. ${ }^{(2)}$ When the local body temperature increases the temperature of the tissue, the blood vessels expand and the capillary blood flows. Consequently, the body temperature is increased via blood circulation. In contrast, when the local temperature of the human body decreases due to the cold, the peripheral blood vessels shrink owing to the excitement of sympathetic nerves and reduced vascular movement. Moreover, the blood flow and the temperature of the skin decrease and the metabolic activity of the body drops.

\subsection{Trends in temperature sensors}

Wearable sensors are fast becoming a key instrument among temperature sensors. In recent years, there has been an increasing amount of literature on flexible and wearable temperature sensors. Different materials, such as stretchable graphene thermistors, ${ }^{(3)}$ organic semiconductors, ${ }^{(4)}$ stretchable polyaniline nanofibers, ${ }^{(5)}$ graphene nanowalls, ${ }^{(6)}$ organic flexible arrays ${ }^{(7)}$ polyethylene terephthalate, ${ }^{(8)}$ silicon Fabry-Pérot cavities, ${ }^{(9)} \mathrm{Er}^{3+}$-doped calcium aluminate glass, ${ }^{(10)}$ and dual-functional nitrogen- and sulfur-codoped carbon dots (C-dots) ${ }^{(11)}$ have been used as temperature sensors. They can be attached to the human $\operatorname{skin}^{(12)}$ to consider the effect of temperature on the mechanical properties of skin or can be added to wireless ECG sensors $^{(13)}$ to transfer data on the temperature of the human body with ECG data. However, major problems with this type of temperature sensor are the strain limitation during mechanical deformation, the need of a prestrained substrate for the fabricated layers, and opacity. In addition, their manufacturing processes are very complex and expensive.

Different technologies for indicating thermal comfort such as air temperature sensors, wristworn devices, and thermal cameras have been compared. ${ }^{(14)}$ A high-tech temperature sensor to measure the temperature of exhaled breath and detect pathogenic progression has been proposed. ${ }^{(15)}$ A flexible wearable thermoelectric nanogenerator made from $\mathrm{Bi}_{2} \mathrm{Te}_{3}$ was proposed for use 
in next-generation wearable temperature sensors; this material can be easily incorporated into textiles. ${ }^{(16)}$ A stretchable temperature sensor that removes strain was developed for use in smart healthcare devices. ${ }^{(17)}$ A portable hypothermia device can be used for hypothermia therapy to maintain a low body temperature in pigs and decrease the body temperature via a thermoelectric element included in the pad part. ${ }^{(18)}$

As mentioned before, the stability and control of temperature are closely associated with certain diseases. The thermoregulation mechanism has been used in many devices to suppress viral disease and maintain the quality of life. A wearable thermoregulation device maintains the body temperature. Thermoregulation was utilized in the thermoelectric devices to decrease the human body temperature. ${ }^{(19)}$ A feedback circuit based on the thermoregulation mechanism to break fever has been proposed. ${ }^{(20)}$ A cold pad device for thermoregulation was used as a body temperature sensor to measure the temperature, and increase and decrease the body temperature using water via a thermoelectric module. This water circulates through the pad and controls the body temperature. Most of these devices were designed to artificially control the whole-body temperature. However, cooling technology has largely remained unchanged for more than a century and it still relies on the cooling of the entire space regardless of the number of occupants. Development of a wearable temperature sensor based on local heating and cooling of the human body is required and still remains a challenge.

In this study, a wearable temperature sensor based on the thermoregulation mechanism that uses a Peltier thermoelectric device was developed to change the human body temperature. The wearable temperature sensor was attached to the wrist using a bracelet, which can monitor, control, and change the temperature of the human body using a local temperature change. The body temperature was changed by transferring the heat from one side to the other side of the Peltier semiconductor or vice versa. The feedback control system was used to maintain the human body temperature during increases and decreases in the surrounding environment temperature. This technology provides a basis for the next generation of wearable temperature sensors to control and change the temperature of the human body.

\section{Materials and Methods}

\subsection{Peltier component}

A Peltier component is a thin plate-shaped device with each side used as a heat source or sink. In a Peltier component, a direct current voltage is applied across two different devices using the thermocouple principle, and the heat is absorbed on one side and generated on the other side according to the direction of the current (Peltier effect). Table 1 provides the electrical properties of the Peltier device used in this study.

Furthermore, the heat is absorbed by the low-temperature side and released on the hightemperature side. Thus, the Peltier component acts as a heat pump that pushes heat from the low-temperature side to the high-temperature side. The heat pump direction and current are changed with the heat. Therefore, the Peltier component acts as a semiconductor element that changes the amount of heat pumped to enable free cooling, heating, and temperature control. 
Table 1

Electrical properties of Peltier device.

\begin{tabular}{lc}
\hline Model name & DMPE-12709 \\
\hline Size $\left(\mathrm{mm}^{3}\right)$ & $40 \times 40 \times 3.5$ \\
Voltage $(\mathrm{V})$ & 12 \\
Maximum voltage (V) & 15 \\
Maximum current (A) & 9 \\
Maximum power consumption (W) & 83.16 \\
$\Delta T_{\max }\left({ }^{\circ} \mathrm{C}\right)$ & $\geq 66$ \\
\hline
\end{tabular}

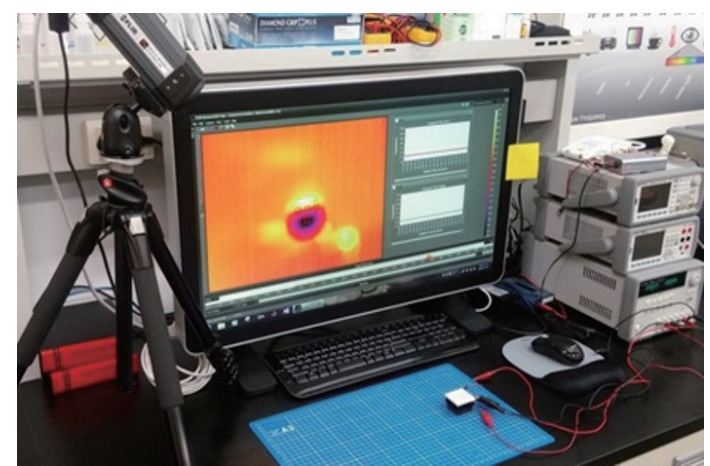

Fig. 3. (Color online) Measurement of temperature in Peltier element.

The Peltier component was utilized in our experiment because of its small size, light weight, the possibility of fabrication to the desired shape, lifetime, and reliability. It responds to temperature changes instantly and includes no parts that cause mechanical fatigue or damage.

We investigated the temperature change of the heat source and heat sink of the Peltier component. The electrodes of the Peltier component were connected to a $12 \mathrm{~V}$ power supply. A FLIR thermal imaging camera and FLIR ResearchIR software were used to measure the rise or fall in temperature owing to the applied voltage. Then, the Peltier component was photographed via the FLIR thermal imaging camera. We used the FLIR ResearchIR program connected to the camera to observe the temperature change of the Peltier component in real time via images, data, and graphs. Figure 3 illustrates the measurement of temperature in the Peltier device using the FLIR thermal imaging camera.

Figures 4(a) and 4(b) show the heat sink of the Peltier component, Fig. 4(c) illustrates the Peltier component without an applied current, and Figs. 4(d) and 4(e) show the heat sources of the Peltier component measured using the infrared camera. When a voltage was applied to the Peltier component, the heat sink temperature dropped from $26-27{ }^{\circ} \mathrm{C}$ to $23.8{ }^{\circ} \mathrm{C}$ and then to $21.9^{\circ} \mathrm{C}$. Meanwhile, the heat source temperature increased from 45.3 to $60.1{ }^{\circ} \mathrm{C}$. The classification algorithm ${ }^{(21)}$ can be utilized to classify increasing and decreasing temperatures.

As shown in Fig. 5, when the voltage was applied, the heat sink absorbed the ambient heat and its temperature dropped below room temperature. In contrast, the heat from the heat sink and the lost electrical energy were dissipated from the heat sink. If the heat in the Peltier component cannot be dissipated, the component will begin to heat up regardless of whether it is a heat source or heat sink. As shown in Fig. 5, even if the temperature of the heat sink initially decreases, the heat sink of a Peltier component without a heat dissipation device gradually increases in temperature owing to the heat source.

Thus, as shown in Fig. 6(b), a heat sink (plate) and fan are attached to dissipate the heat source to the surroundings and to dissipate the heat of the Peltier element and decrease the temperature of the heat sink to below $15^{\circ} \mathrm{C}$. 


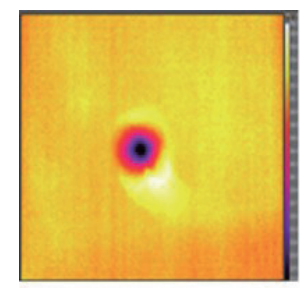

(a)

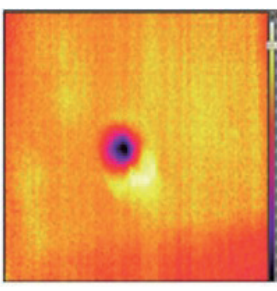

(b)

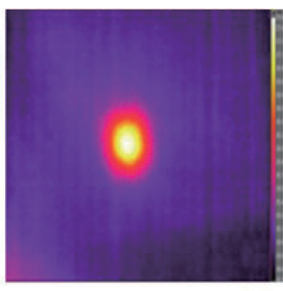

(c)

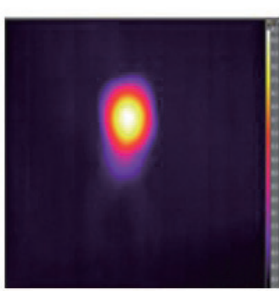

(d)

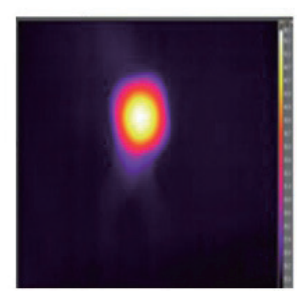

(e)

Fig. 4. (Color online) Heat absorption and generation of Peltier component photographed through FLIR thermal imaging camera. (a) and (b) Heat sinks of the Peltier component, (c) Peltier component without an applied current, (d) and (e) heat source of the Peltier component measured using an infrared camera.

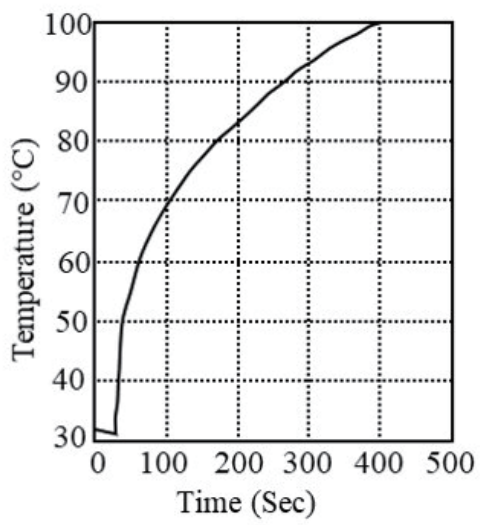

Fig. 5. Temperature of area heated using Peltier element plotted against time.

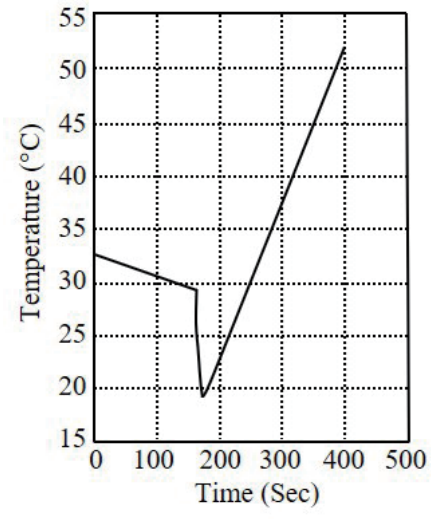

(a)

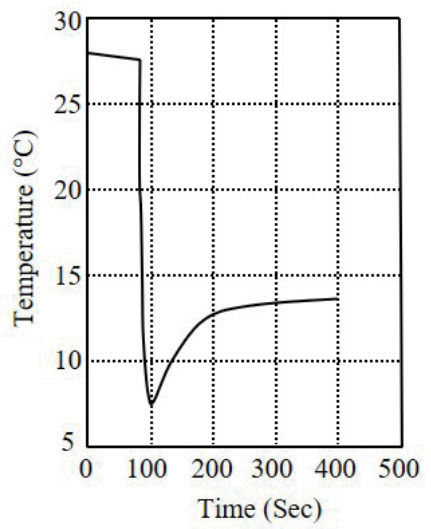

(b)

Fig. 6. (a) Heat absorption and (b) heat generation of the Peltier device. 


\subsection{Additive liquid silicone (Dow Corning SYLGARD ${ }^{\text {TM }}$ 184)}

SYLGARD ${ }^{\mathrm{TM}} 184$ can be used for quick and adaptive cure processing owing to its good dielectric properties, flowability, and high transparency. It can be used as a coating and a molding agent, a lubricant, and a softener. In our experiment, we weighed and mixed SYLGARD ${ }^{\text {TM }} 184$ to coat the Peltier component via a curing agent (ratio of 10:1), and we stirred the mixture for $20 \mathrm{~min}$ to form sufficient bubbles and make the mixture uniform. Then, we fixed the Peltier component in a slightly floating position in a rectangular plastic container that could be fit to the element, and finally poured the SYLGARD ${ }^{\mathrm{TM}} 184$ into the container. We removed the bubbles and placed the container in a PURIVEN drying oven for $20 \mathrm{~min}$ at $125^{\circ} \mathrm{C}$. The silicon was placed on the Peltier component as shown in Fig. 7.

\subsection{Feedback control system}

The feedback control or closed-loop control system refers to the reference input, which is compared with the output using a feedback control system to reach a desired value. The output of the system $Y(s)$ is returned to the summing point and compared with the reference input $X(s)$. The feedback control system is illustrated in Fig. 8. $Y(s)$ is found by multiplying the transfer function $G(s)$ by the input to the system $E(s)$. The output is converted and modified using the feedback element $H(s)$ then compared with the input signal. The closed-loop transfer function relating $X(s)$ to $Y(s)$ is calculated using

$$
Y(s)=G(s) /(1+G(s) H(s)) X(s) .
$$

One of the feedback control systems in nature is the human body. The feedback control system is used to maintain the human body temperature during increases and decreases in the temperature of the environment. By measuring the real body temperature and comparing it with a constant human body temperature as a desirable temperature, the Peltier device changes the current direction and the heating or cooling sides in such a way as to guarantee that the body temperature remains at a desirable level regardless of the environmental temperature.

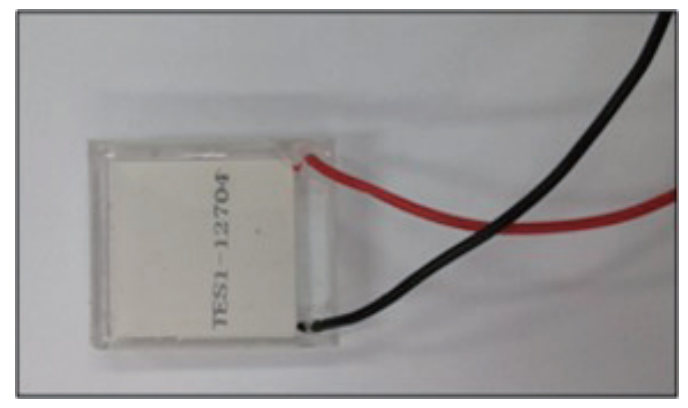

Fig. 7. (Color online) Peltier component coated with SYLGARD ${ }^{\mathrm{TM}} 184$.

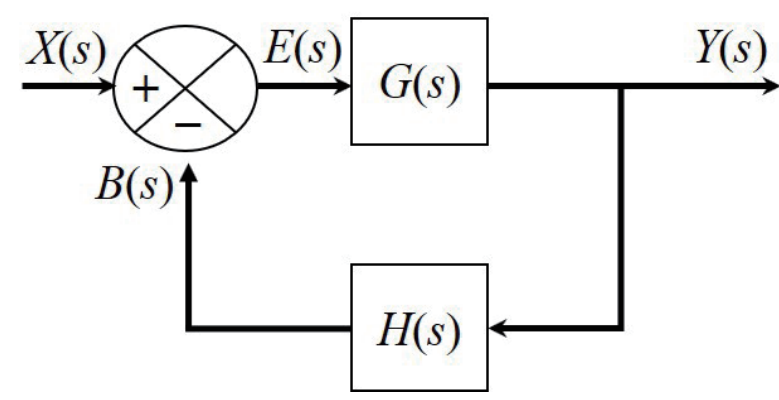

Fig. 8. Feedback control system of the Peltier element. 
To control the body temperature, the output signal is the constant body temperature. The output temperature is converted to a voltage and compared with the desirable temperature. $Y(s)$ and $H(s)$ are the body and sensor temperatures, respectively. When the body temperature starts to rise, it is detected by the temperature sensor, and the desired temperature $X(s)$ is continuously input while the wearable thermoregulation device is controlled by the temperature sensor until the body temperature reaches $X(s)$.

\subsection{Wearable thermoregulation device}

In this study, a wearable sensor was developed as a bracelet-type wearable device. A Peltier component was used to artificially control the local temperature to induce changes in the human body temperature. Then the temperature sensor was directly attached to the human body to measure the current body temperature, and the data were transferred to a motherboard to compare the reference temperature with the Peltier device temperature. A TMP 102 digital temperature sensor was used to measure the body temperature, which has an accuracy of $0.5^{\circ} \mathrm{C}$ from -25 to $85{ }^{\circ} \mathrm{C}$. Furthermore, a lithium battery was used for the power supply and a motherboard was attached to either side of the Peltier component. A small Arduino Mini was used to attach the motherboard to the wrist.

\subsubsection{Control of Peltier component using Arduino}

An Arduino Uno board and an n-channel MOSFET were connected to a $10 \mathrm{k} \Omega$ resistance using a breadboard. The Peltier device requires a $15.4 \mathrm{~V}, 7$ A power supply. While a PWM port with pin Nos. 3, 5, 6, 9, 10, and 11 can be used to control the current, pin No. 3 was used in this experiment. We connected it to the breadboard and input it to the Arduino. We opened the serial monitor and defined the letter "a" for an increment of the power supply to $5 \mathrm{~V}$. By defining "a" again, the power was increased to $10 \mathrm{~V}$. By entering " $\mathrm{z}$ ", it was decreased to $5 \mathrm{~V}$.

\subsubsection{Control of Peltier component using Arduino conceptual diagram of thermoregulation devices}

The wearable thermoregulation device increases or decreases the body temperature according to temperature changes to maintain it at a fixed level. The thermoregulation device consists of a sensor for measuring the human body temperature, cooling and heating parts that contain a thermoelectric module that switches between cooling and heating according to the polarity of the applied power supply, a control part for controlling the operation of the cooling and heating parts according to the desired body temperature, and a sensor that measures the body temperature. If the body temperature measured by the sensor is greater than the desired body temperature, then the designed device on the research participant's wrist operates in the temperature-lowering mode and attempts to reduce the body temperature to the desired level. If the body temperature is less than the desired level, then the device operates in the temperatureraising mode, and it begins its heating operation to increase the body temperature to the desired level. 


\section{Results and Discussion}

The wearable sensor was used to induce an increase or decrease in the local body temperature, resulting in a change in the overall body temperature. We performed experiments on humans with informed consent, and the wearable sensor was composed of commercial components that can be used for humans. First, the initial human body temperature was measured to be $37.5{ }^{\circ} \mathrm{C}$ (which is the normal human body temperature) before using the wearable sensor. Then, the body temperature was raised by the surrounding environment, and the wearable sensor responded by displaying a 'cold' sign, which was used to induce a decrease in body temperature. In contrast, when the body temperature was decreased by the surrounding environment, the wearable sensor responded by displaying a 'hot' sign, which was used to induce an increase in body temperature. The average change was $0.4-0.6{ }^{\circ} \mathrm{C}$ from 32.1 to $32.6{ }^{\circ} \mathrm{C}$. By comparing the temperature change with and without wearing the wearable sensor, it was confirmed that the Peltier device can increase and decrease the body temperature. Figure 9 shows changes in the body temperature over $300 \mathrm{~s}$ in 100 trials as relative increases and decreases. The highest increase was $86.1 \%$ with a change from 18.7 to $34.8{ }^{\circ} \mathrm{C}$, the lowest increase was $1.2 \%$ with a change from 24.6 to $24.9{ }^{\circ} \mathrm{C}$, and the average increase was $33.5 \%$ with a standard deviation of $25.5 \%$. The highest decrease was $3.75 \%$ with a change from 34.1 to $32.9{ }^{\circ} \mathrm{C}$, the lowest decrease was $0.6 \%$ with a change from 34.7 to $34.5{ }^{\circ} \mathrm{C}$, and the average decrease was $1.6 \%$ with a standard deviation of $0.7 \%$. The data shows that when the body

(a)

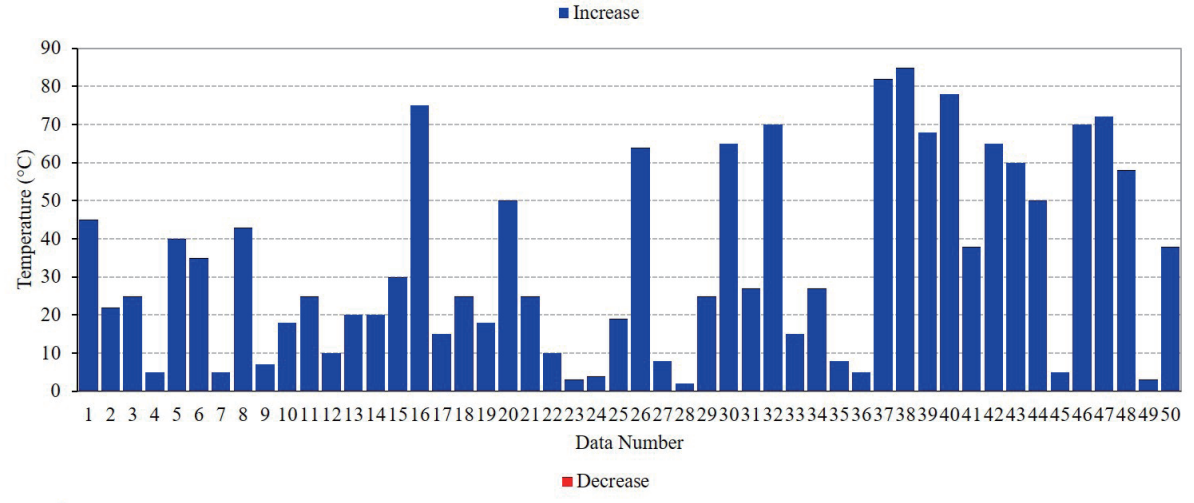

(b)

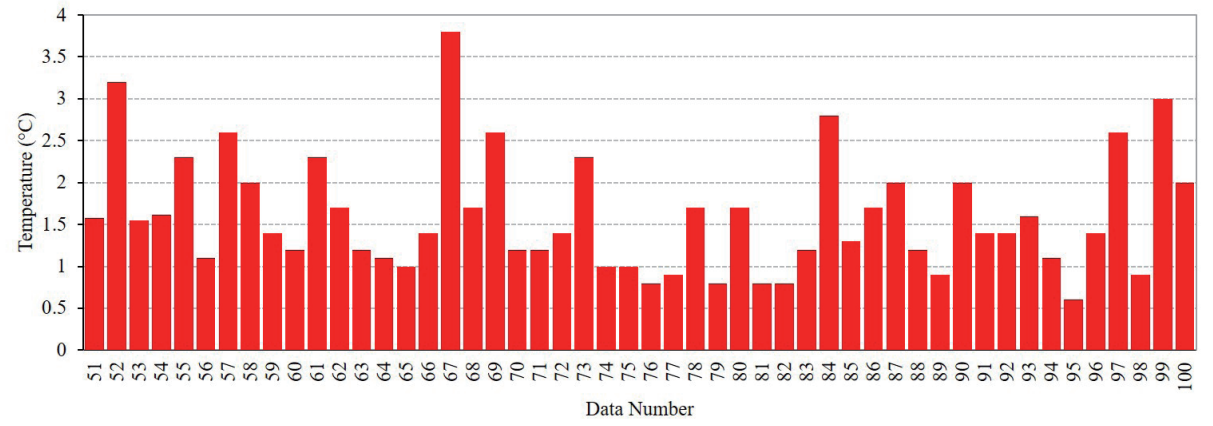

Fig. 9. (Color online) Changes in body temperature over $300 \mathrm{~s}$ in 100 trials: (a) relative temperature increase and (b) relative temperature decrease. 


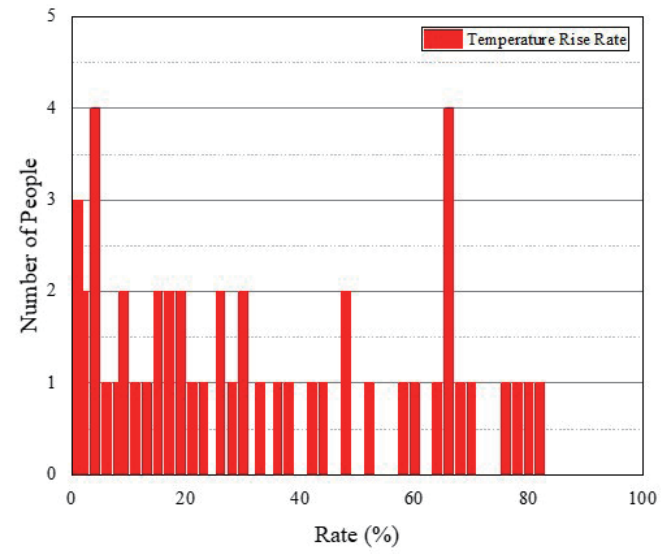

(a)

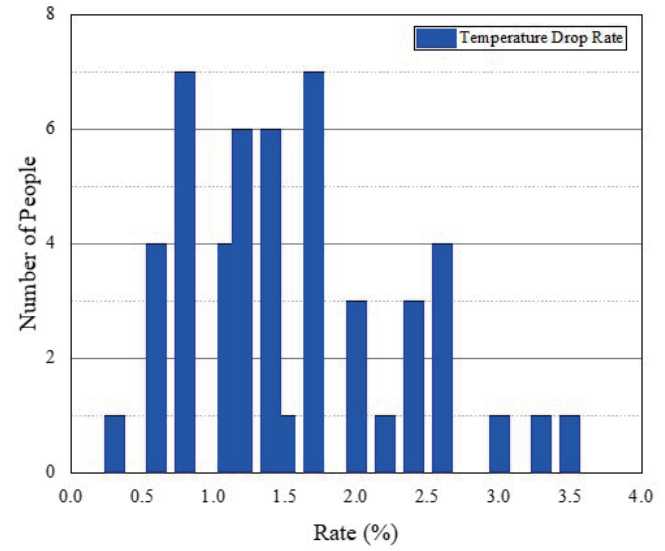

(b)

Fig. 10. (Color online) Distributions of (a) relative increase and (b) relative decrease in temperature.

temperature was abnormally low, there was a large relative change due to heating of a local area by the Peltier device, which quickly returned the temperature to normal.

The large standard deviation of the relative increase shown in Fig. 10(a) is considered to be due to the large difference between the initial body temperature and the subject's ability to produce thermoregulatory heat. On the other hand, the low relative change in the test where the body temperature was decreased appears to have been initiated at a temperature close to the average normal body temperature because the subjects did not abnormally increase their starting temperature before the test. The wearable sensor was operated to drop the body temperature to a normal value; it would not have dropped significantly owing to the nature of heat generation in a body to maintain the body temperature. As shown in Fig. 10(b), the standard deviation of the relative increase was not large owing to the similar starting temperature, and although the relative change was small, it can be seen that the temperature dropped in each case owing to the wearable sensor.

The abnormally elevated body temperature confirmed that the subject's body temperature was less than the temperature controlled by the hypothalamus. The difference in the subject's perceived temperature due to the heat generated by the wearable sensor needs attention. Some subjects felt lukewarm when they heated their wrists in the first experiment, whereas others felt hot. Many subjects felt cold in the second experiment. Depending on the subject's perceived temperature, the user may need to manually change the temperature.

\section{Conclusions}

A wearable temperature sensor based on a Peltier thermoelectric device was proposed. The sensor was attached to the wrist using a bracelet and can monitor, control, and change the human body temperature using a local temperature change. The body temperature was changed by transferring the heat from one side to the other side of the Peltier semiconductor. The 
experimental results indicated that the changes in the body temperature were small, although a change did occur. The highest relative increase in temperature was $86.1 \%$ with a change from 18.7 to $34.8^{\circ} \mathrm{C}$, while the lowest relative increase was $1.2 \%$ with a change from 24.6 to $24.9{ }^{\circ} \mathrm{C}$, with an average change of $33.5 \%$ and a standard deviation of $25.5 \%$. This technology provides a basis for next-generation wearable temperature sensors to control and change the temperature of the human body. Also, these sensors can be used to help elderly patients change and control their body temperature.

\section{Acknowledgments}

This research was supported by Korea Basic Science Institute (National Research Facilities and Equipment Center) grant funded by the Ministry of Education (grant No. 2020R1A6C1020263).

\section{References}

1 F. Emami, A. Banstola, A. Vatanara, S. Lee, J. O. Kim, J.-H. Jeong, and S. Yook: Mol. Pharm. 16 (2019) 1184. https://doi.org/10.1021/acs.molpharmaceut.8b01157

2 R. L. Myers: The Basics of Chemistry (Greenwood Publishing Group, 2003) p. 392. https://products.abc-clio. com/ABC-CLIOCorporate/product.aspx?pc $=$ B1610P

3 C. Yan, J. Wang, and P. S. Lee: ACS nano 9 (2015) 2130. https://doi.org/10.1021/nn507441c

4 T. Someya, Y. Kato, T. Sekitani, S. Iba, Y. Noguchi, Y. Murase, H. Kawaguchi, and T. Sakurai: Proc. Natl. Acad. Sci. 102 (2005) 12321. https://doi.org/10.1073/pnas.0502392102

5 S. Y. Hong, Y. H. Lee, H. Park, S. W. Jin, Y. R. Jeong, J. Yun, I. You, G. Zi, and J. S. Ha: Adv. Mater. 28 (2016) 930. https://doi.org/10.1002/adma.201504659

6 J. Yang, D. Wei, L. Tang, X. Song, W. Luo, J. Chu, T. Gao, H. Shi, and C. Du: RSC Adv. 5 (2015) 25609. https://doi.org/10.1039/C5RA00871A

7 X. Ren, K. Pei, B. Peng, Z. Zhang, Z. Wang, X. Wang, and P. K. Chan: Adv. Mater. 28 (2016) 4832. https://doi. org/10.1002/adma.201600040

8 Y. Yamamoto, D. Yamamoto, M. Takada, H. Naito, T. Arie, S. Akita, and K. Takei: Adv. Healthcare Mater. 6 (2017) 1700495. https://doi.org/10.1002/adhm.201700495

9 G. Liu, M. Han, and W. Hou: Opt. Express 23 (2015) 7237. https://doi.org/10.1364/OE.23.007237

10 S. F. León-Luis, V. Monteseguro, U. R. Rodríguez-Mendoza, I. R. Martín, D. Alonso, J. M. Cáceres, and V. Lavín: J. Lumin. 179 (2016) 272. https://doi.org/10.1016/j.jlumin.2016.07.005

11 X. Cui, Y. Wang, J. Liu, Q. Yang, B. Zhang, Y. Gao, Y. Wang, and G. Lu: Sens. Actuators, B 242 (2017) 1272. https://doi.org/10.1016/j.snb.2016.09.032

12 S. J. M. Yazdi, K. S. Cho, and N. Kang: Korea-Aust. Rheol. J. 30 (2018) 293. https://doi.org/10.1007/s13367018-0027-5

13 S. J. Mostafavi Yazdi and J. Lee: Basic Clini. Pharmacol. Toxicol. 125 (2019) 23. https://oi.org/10.1111/ bcpt.13326

14 A. Aryal and B. Becerik-Gerber: Build. Environ. 160 (2019) 106223. https://doi.org/10.1016/ j.buildenv.2019.106223

15 J. Shin, B. Jeong, J. Kim, V. B. Nam, Y. Yoon, J. Jung, S. Hong, H. Lee, H. Eom, and J. Yeo: Adv. Mater. 32 (2019) 1905527. https://doi.org/10.1002/adma.201905527

16 R. Feng, F. Tang, N. Zhang, and X. Wang: ACS Appl. Mater. Interfaces 11 (2019) 38616. https://doi. org/10.1021/acsami.9b11435

17 T. Q. Trung, T. M. L. Dang, S. Ramasundaram, P. T. Toi, S. Y. Park, and N.-E. Lee: ACS Appl. Mater. Interfaces 11 (2018) 2317. https://doi.org/10.1021/acsami.8b19425

18 E. Rabenstein, M. Tyree, D. Dirnberger, and R. DiGeronimo: J Neonatal Med. 3 (2010) 15.

19 S. Hong, Y. Gu, J. K. Seo, J. Wang, P. Liu, Y. S. Meng, S. Xu, and R. Chen: Sci. Adv. 5 (2019) eaaw0536. https://doi.org/10.1126/sciadv.aaw0536

20 B. Duan and X. S. Xu: Neuron 103 (2019) 179. https://doi.org/10.1016/j.neuron.2019.06.023 
21 S. Yang, H.-J. Yoon, S. J. M. Yazdi, and J.-H. Lee: Int. J. Med. Robot. 16 (2020) e2033. https://doi.org/10.1002/ res. 2033

\section{About the Authors}

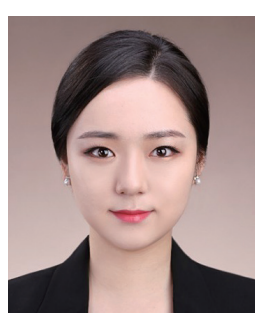

Eun-bin Park received her B.S. and M.S. degrees from Keimyung University, Daegu, South Korea, in 2016 and 2019, respectively. She will be a Ph.D. student from 2020 at the Artificial Intelligence and Computed Diagnosis Lab (AICD), Biomedical Engineering Department, Keimyung University. Her research interest is in biomedical sensors. (peb0822@naver.com)

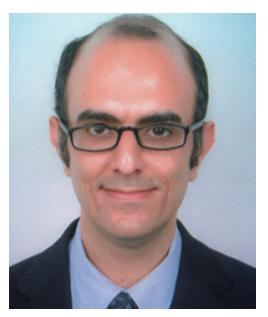

Seyed Jamaleddin Mostafavi Yazdi received his B.Sc. degree in mechanical engineering in 2005 from Yazd University, Yazd, Iran, his M.Sc. degree in aerospace engineering in 2009 from K.N. Toosi University of Technology, Tehran, Iran, and his Ph.D. degree in mechanical engineering from Kyungpook National University, Daegu, South Korea, in 2019. From March 2019 to February 2020, he was a postdoctoral researcher at the Artificial Intelligence and Computed Diagnosis Lab (AICD), Biomedical Engineering Department, Keimyung University, Daegu, South Korea. Currently, he is a visiting scholar at NVH and Experimental Mechanics Laboratory, Department of Mechanical Engineering, Kettering University, Flint, MI, USA. His research interests lie in mechanical engineering (biomechanics) and biomedical engineering, focusing on implantable electrocardiogram (ECG) sensors and signal characterization of ECG signals for atrial fibrillation using machine learning. (smostafaviyazdi@kettering.edu)

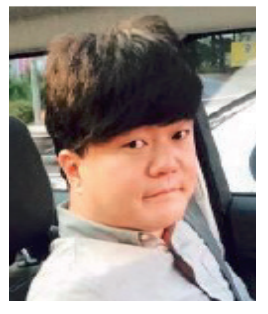

Jong-Ha Lee received his B.S. degree in electronics engineering in 2000 from Inha University, Incheon, South Korea, his M.S. degree in electrical engineering in 2005 from New York University, Brooklyn, New York, USA, and his Ph.D. degree in electrical engineering from Temple University, Philadelphia, PA, USA. He was with Samsung Advanced Institute of Technology as a research staff member. He is currently an associate professor with the Department of Biomedical Engineering, School of Medicine, Keimyung University, Daegu, Korea. His current research interests include tactile sensation imaging for tissue characterization, computer-aided diagnosis, medical image analysis, pattern recognition, and machine learning. (segeberg@kmu.ac.kr) 